Network Working Group

Request for Comments: 2033

Category: Informational

J. Myers

Carnegie Mellon

October 1996

Local Mail Transfer Protocol

Status of this Memo

This memo provides information for the Internet community. This memo does not specify an Internet standard of any kind. Distribution of this memo is unlimited.

\title{
1. Abstract
}

SMTP [SMTP] [HOST-REQ] and its service extensions [ESMTP] provide a mechanism for transferring mail reliably and efficiently. The design of the SMTP protocol effectively requires the server to manage a mail delivery queue.

In some limited circumstances, outside the area of mail exchange between independent hosts on public networks, it is desirable to implement a system where a mail receiver does not manage a queue. This document describes the LMTP protocol for transporting mail into such systems.

Although LMTP is an alternative protocol to ESMTP, it uses (with a few changes) the syntax and semantics of ESMTP. This design permits LMTP to utilize the extensions defined for ESMTP. LMTP should be used only by specific prior arrangement and configuration, and it MUST NOT be used on TCP port 25.

Table of Contents

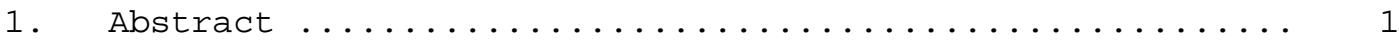

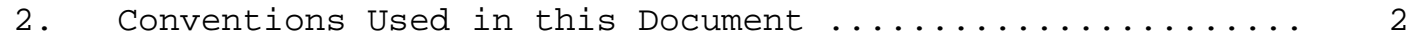

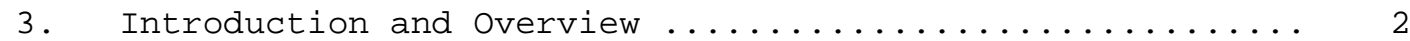

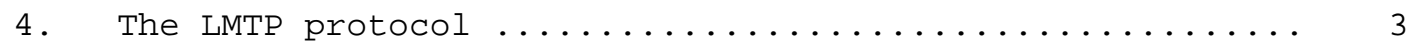

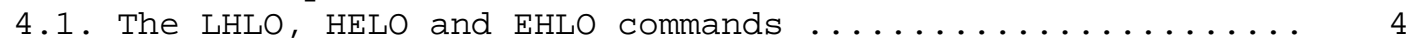

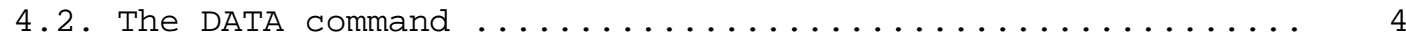

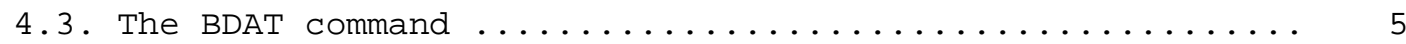

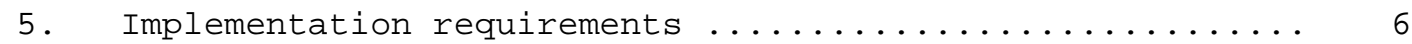

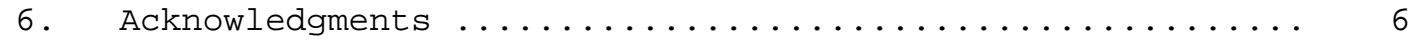

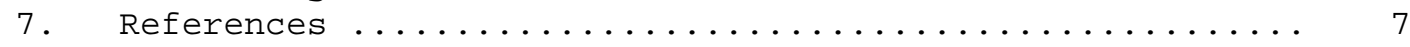

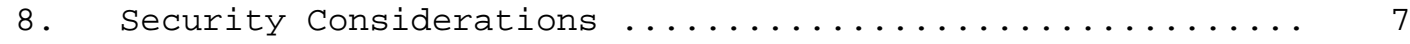

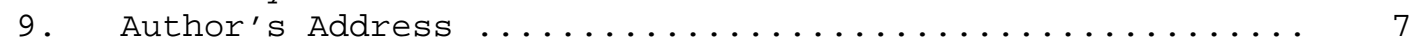


2. Conventions Used in this Document

In examples, "C:" and "S:" indicate lines sent by the client and server respectively.

3. Introduction and Overview

The design of the SMTP protocol effectively requires the server to manage a mail delivery queue. This is because a single mail transaction may specify multiple recipients and the final "." of the DATA command may return only one reply code, to indicate the status of the entire transaction. If, for example, a server is given a transaction for two recipients, delivery to the first succeeds, and delivery to the second encounters a temporary failure condition, there is no mechanism to inform the client of the situation. The server must queue the message and later attempt to deliver it to the second recipient.

This queuing requirement is beneficial in the situation for which SMTP was originally designed: store-and-forward relay of mail between networked hosts. In some limited situations, it is desirable to have a server which does not manage a queue, instead relying on the client to perform queue management. As an example, consider a hypothetical host with a mail system designed as follows:

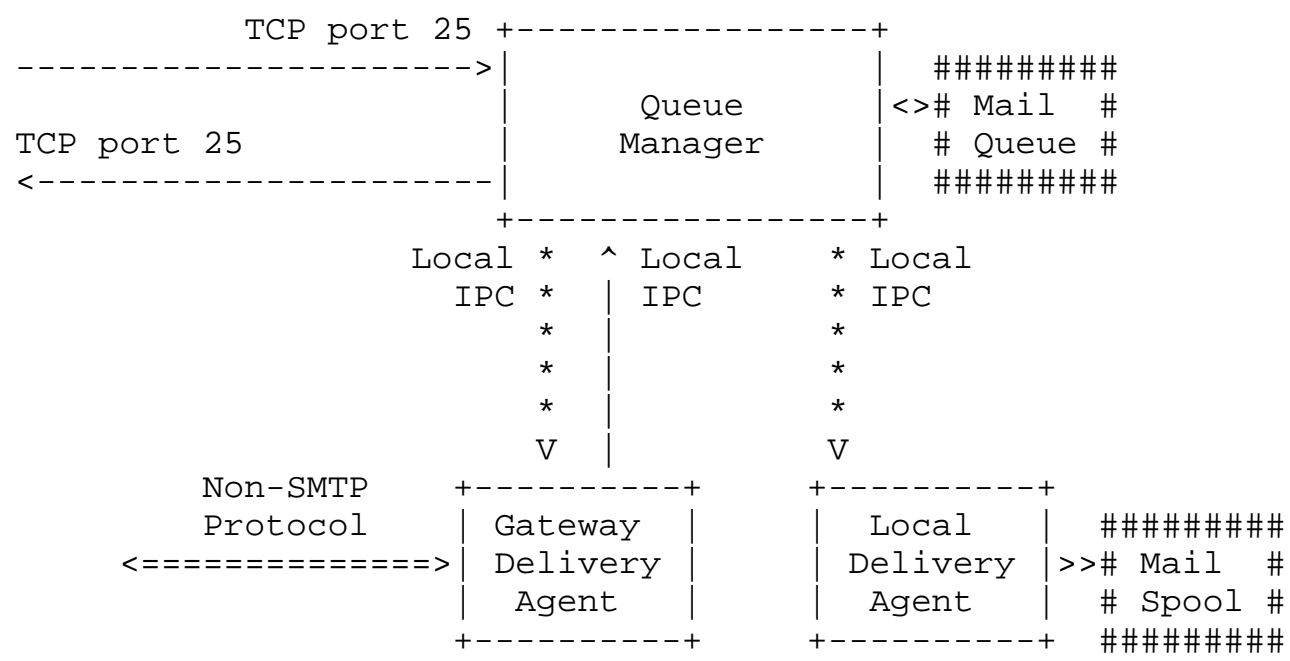

The host's mail system has three independent, communicating subsystems. The first is a queue manager, which acts as a 
traditional SMTP agent, transferring messages to and from other hosts over TCP and managing a mail queue in persistent storage. The other two are agents which handle delivery for addresses in domains for which the host takes responsibility. One agent performs gatewaying to and from some other mail system. The other agent delivers the message into a persistent mail spool.

It would be desirable to use SMTP over a local inter-process communication channel to transfer messages from the queue manager to the delivery agents. It would, however, significantly increase the complexity of the delivery agents to require them to manage their own mail queues.

The common practice of invoking a delivery agent with the envelope address(es) as command-line arguments, then having the delivery agent communicate status with an exit code has three serious problems: the agent can only return one exit code to be applied to all recipients, it is difficult to extend the interface to deal with ESMTP extensions such as DSN [DSN] and ENHANCEDSTATUSCODES [ENHANCEDSTATUSCODES], and exits performed by system libraries due to temporary conditions frequently get interpreted as permanent errors.

The LMTP protocol causes the server to return, after the final "." of the DATA command, one reply for each recipient. Therefore, if the queue manager is configured to use LMTP instead of SMTP when transferring messages to the delivery agents, then the delivery agents may attempt delivery to each recipient after the final "." and individually report the status for each recipient. Connections which should use the LMTP protocol are drawn in the diagram above using asterisks.

Note that it is not beneficial to use the LMTP protocol when transferring messages to the queue manager, either from the network or from a delivery agent. The queue manager does implement a mail queue, so it may store the message and take responsibility for later delivering it.

4. The LMTP protocol

The LMTP protocol is identical to the SMTP protocol SMTP [SMTP] [HOST-REQ] with its service extensions [ESMTP], except as modified by this document.

A "successful" RCPT command is defined as an RCPT command which returns a Positive Completion reply code.

A "Positive Completion reply code" is defined in Appendix E of STD 10, RFC 821 [SMTP] as a reply code which "2" as the first digit. 


\subsection{The LHLO, HELO and EHLO commands}

The HELO and EHLO commands of ESMTP are replaced by the LHLO command. This permits a misconfiguration where both parties are not using the same protocol to be detected.

The LHLO command has identical semantics to the EHLO command of ESMTP [ESMTP ].

The HELO and EHLO commands of ESMTP are not present in LMTP. A LMTP server MUST NOT return a Postive Completion reply code to these commands. The 500 reply code is recommended.

\subsection{The DATA command}

In the LMTP protocol, there is one additional restriction placed on the DATA command, and one change to how replies to the final "." are sent.

The additional restriction is that when there have been no successful RCPT commands in the mail transaction, the DATA command MUST fail with a 503 reply code.

The change is that after the final ".", the server returns one reply for each previously successful RCPT command in the mail transaction, in the order that the RCPT commands were issued. Even if there were multiple successful RCPT commands giving the same forward-path, there must be one reply for each successful RCPT command.

When one of these replies to the final "." is a Positive Completion reply, the server is accepting responsibility for delivering or relying the message to the corresponding recipient. It must take this responsibility seriously, i.e., it MUST NOT lose the message for frivolous reasons, e.g., because the host later crashes or because of a predictable resource shortage.

A multiline reply is still considered a single reply and corresponds to a single RCPT command.

\section{EXAMP LE :}

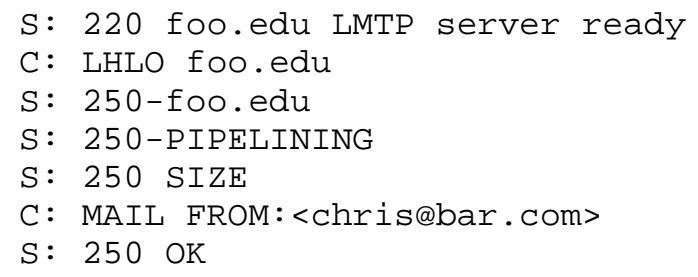




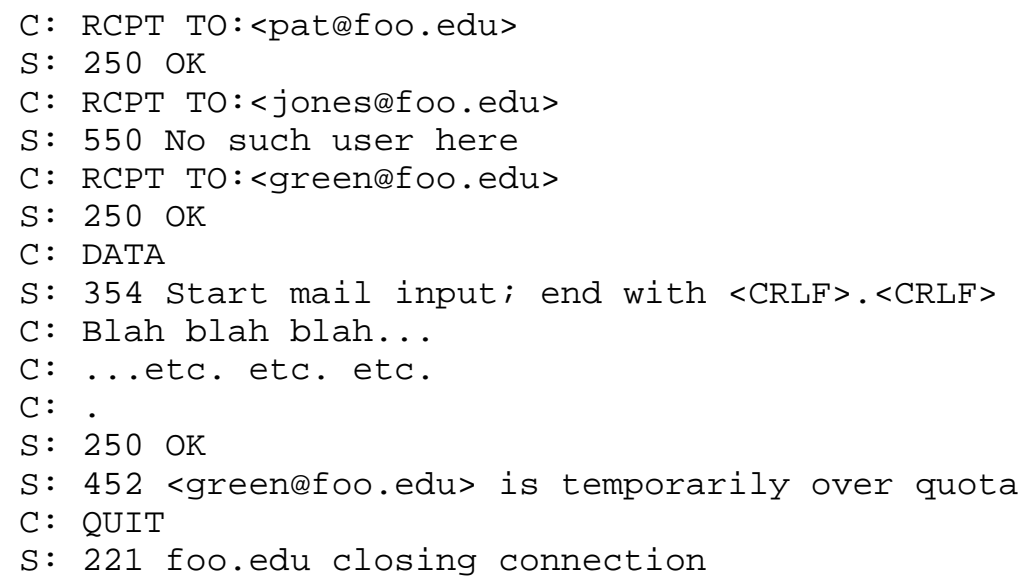

NOTE: in the above example, the domain names of both the client and server are identical. This is because in the example the client and server are different subsystems of the same mail domain.

\subsection{The BDAT command}

If the server supports the ESMTP CHUNKING extension [BINARYMIME], a BDAT command containing the LAST parameter returns one reply for each previously successful RCPT command in the mail transaction, in the order that the RCPT commands were issued. Even if there were multiple successful RCPT commands giving the same forward-path, there must be one reply for each successful RCPT command. If there were no previously successful RCPT commands in the mail transaction, then the BDAT LAST command returns zero replies.

When one of these replies to the BDAT LAST command is a Positive Completion reply, the server is accepting responsibility for delivering or relaying the message to the corresponding recipient. It must take this responsibility seriously, i.e., it MUST NOT lose the message for frivolous reasons, e.g., because the host later crashes or because of a predictable resource shortage.

A multiline reply is still considered a single reply and corresponds to a single RCPT command.

The behavior of BDAT commands without the LAST parameter is not changed; they still return exactly one reply. 
5. Implementation requirements

As LMTP is a different protocol than SMTP, it MUST NOT be used on the TCP service port 25 .

A server implementation MUST implement the PIPELINING [PIPELINING] and ENHANCEDSTATUSCODES [ENHANCEDSTATUSCODES] ESMTP extensions. A server implementation SHOULD implement the 8BITMIME [8BITMIME] extension.

Use of LMTP can aggravate the situation described in [DUP-MSGS]. To avoid this synchronization problem, the following requirements are made of implementations:

A server implementation which is capable of quickly accepting responsibility for delivering or relaying a message to multiple recipients and which is capable of sending any necessary notification messages SHOULD NOT implement the LMTP protocol.

The LMTP protocol SHOULD NOT be used over wide area networks.

The server SHOULD send each reply as soon as possible. If it is going to spend a nontrivial amount of time handling delivery for the next recipient, it SHOULD flush any outgoing LMTP buffer, so the reply may be quickly received by the client.

The client SHOULD process the replies as they come in, instead of waiting for all of the replies to arrive before processing any of them. If the connection closes after replies for some, but not all, recipients have arrived, the client MUST process the replies that arrived and treat the rest as temporary failures.

6. Acknowledgments

This work is a refinement of the MULT extension, which was invented by Jeff Michaud and was used in implementing gateways to the Mail-11 mail system.

Many thanks to Matt Thomas for assisting me in understanding the semantics of the Mail-11 MULT extension. 
7. References

[8BITMIME] Klensin, J., et. al, "SMTP Service Extension for 8bit-MIME transport", RFC 1652, July 1994.

[BINARYMIME] Vaudreuil, G., "SMTP Service Extensions for Transmission of Large and Binary MIME Messages", RFC 1830, August 1995.

[DSN] Moore, K., Vaudreuil, G., "An Extensible Message Format for Delivery Status Notifications", RFC 1894, January 1996.

[DUP-MSGS] Partridge, C., "Duplicate messages and SMTP", RFC 1047, February 1988 .

[ENHANCEDSTATUSCODES] Freed, N., "SMTP Service Extension for Returning Enhanced Error Codes", RFC 2034, October 1996.

[ESMTP] Rose, M., Stefferud, E., Crocker, C., Klensin, J., Freed, N., "SMTP Service Extensions", RFC 1869, November 1995.

[HOST-REQ] Braden, R., "Requirements for Internet hosts - application and support", STD 3, RFC 1123 section 5, October 1989.

[PIPELINING] Freed, N., Cargille, A, "SMTP Service Extension for Command Pipelining", RFC 1854, October 1995.

[SMTP] Postel, J., "Simple Mail Transfer Protocol", STD 10, RFC 821, August 1982 .

There are no known security issues with the issues in this memo.

9. Author's Address

John G. Myers

Carnegie-Mellon University

5000 Forbes Ave.

Pittsburgh PA, 15213-3890

EMail: jgm+@cmu.edu 